Original Article

\title{
PREVALENCE OF CANDIDA ASSOCIATED DENTURE STOM ATITIS (CADS) AND SPECIATION OF CANDIDA AMONG COM PLETE DENTURE WEARERS OF SOUTH WEST COASTAL REGION OF KARNATAKA
}

\author{
Vinaya Bhat ${ }^{1}$, S.M. Sharma ${ }^{2}$, Veena Shetty ${ }^{3}$, C.S. Shastry ${ }^{4}$, Vaman Rao ${ }^{5}$, \\ Shilpa M. Shenoy ${ }^{6}$, Santanu Saha ${ }^{7} \&$ Sriram Balaji ${ }^{8}$ \\ ${ }^{1} \mathrm{PhD}$ Research Scholar, Department of Prosthodontics, ${ }^{2}$ Professor and HOD, PhD Guide, Oral \& M axillofacial Surgery, \\ ${ }^{3} \mathrm{Co}$-Guide, Department of Microbiology, ${ }^{4} \mathrm{Co}$-Guide \& Principal, ${ }^{5} \mathrm{Co}$-Guide, Department of Biosciences, ${ }^{6}$ Department of \\ M icrobiology, ${ }^{7}$ Senior Lecturer, Department of Pharmacognosy, ${ }^{8}$ Department of Prosthodontics \\ ${ }^{1,2,8}$ A. B. Shetty Memorial Institute of Dental Sciences, ${ }^{3,6} \mathrm{~K}$. S. Hegde Medical Academy, Deralakatte, ${ }^{4,7} \mathrm{NGSM}$ Institute of \\ Pharmaceutical Sciences, Paneer, ${ }^{5}$ NM AM Institute of Technology, NITTE, Mangalore

\section{Correspondence: \\ Vinaya Bhat} \\ Ph.D. Research Scholar, Department of Prosthodontics \\ A. B. Shetty M emorial Institute of Dental Sciences, Deralakatte,Nitte University M angalore 575 018, Karnataka, India \\ Mobile: +9194819 21180, E-mail : drvinayabhat@gmail.com
}

\begin{abstract}
:
A denture wearer is highly prone to develop Candida associated denture stomatitis (CADS), due to the conversion of the normal oral commensal Candida spp. into a pathogen under favorable conditions. Immuno-compromised status, trauma from the prosthesis, other systemic conditions and improper maintenance of the dentures by the patient are few of the causative agents which turn the oral balance into an unhealthy and unsuitable foundation for the wearing of the prosthesis. Prevalence of denture stomatitis caused by the fungi Candida has been identified worldwide and has been reported to be around 65 to $70 \%$ of the denture wearers. The main species that causes infection has been Candida albicans, however, recently, there is a shift to non-Candida albicans-Candida (NCAC).

This cross-sectional survey was undertaken to find the prevalence of denture stomatitis and the causative species in the south westcoastal region of Karnataka.
\end{abstract}

Keywords: Candida-Associated-Denture Stomatitis, Candida species, CHROM agar, Speciation

Introduction:

Infection due to Candida species and other fungi have increased dramatically in recent years and are of prime importance because of the rising number of immunocompromised patients. Although Candida albicans remains the most common cause of infection, the frequency attributed to other members of the genus is also increasing. This is due to the increase in the number of at-

\begin{tabular}{|c|}
\hline Access this article online \\
\hline Quick Response Code \\
\hline
\end{tabular}

risk individuals, particularly those with impaired immunity, such as transplant recipients, cancer patients receiving chemotherapy, and human immunodeficiency virus- infected patients $^{1-16}$.

In the 1980 s C. albicans accounted for more than $80 \%$ of all Candidal isolates recovered from nosocomial yeast infection. Of late, the emergence of Candida species other than $C$. albicans is a matter of concern in several major institutions. These species are also shown to have reduced susceptibility to antifungal agents. The frequency of isolation of Candida krusei, Candida glabrata, Candida tropicalis, and Candida parapsilosis is steadily increasing globally.

In the oral cavity Candida is a normal commensal along with other microbiota. In an immunocompromised patient who is wearing a complete denture, there is a risk of development of Candida associated denture stomatitis 
(CADS) (Pic.01). This is due to the conversion of the normal commensal to an infection causing pathogen. Various factors enhance development and progression of the disease resulting in CADS. ${ }^{1,3,12,15,17-20}$. The prosthesis acts as a foci and trauma from the denture will facilitate infection. Several species of Candida have known to be involved in the process. Even though Candida albicans is the most frequent species, recently, non albicans species have been reported to be dominant ${ }^{21,22}$. Hence, identification of the causative species is essential for the rapid treatment initiation with an appropriate anti fungal agent. There are many techniques to identify Candida species, but among them a technique using CHROMagar Candida (Himedia, M umbai) can easily identify different species of Candida on the basis of colony colour and morphology 23,24 .

This survey was undertaken

a) To estimate the prevalence of Candida associated denture stomatitis in denture wearing patients.

b) To identify the prevalent species of Candida genus in these patients.

\section{Methodology:}

\section{Patient selection}

Fifty five completely edentulous patients (34 male and 21 female) wearing complete denture prosthesis at least for the past one year were randomly selected from the outpatient section of the department of Prosthodontics and KSHEMA, Mangalore. Case history was taken and a structured questionnaire for data collection was filled by the operator after consultation with the patient. Patient's consent to participate in the study was obtained. Ethical clearance was obtained from the ethical committee of NITTEUniversity.

\section{Inclusion criteria:}

1. Age group 50-70 years, male or female patients.

2. Patients wearing complete denture prosthesis.

3. Patients wearing the prosthesis for more than a year.

4. Patients not on any antifungal medication.

5. Patients who are willing to participate in the study.

\section{Exclusion criteria:}

1. Age less than 50 and more than 70 years.
2. Patients not wearing complete denture prosthesis.

3. Patients wearing the prosthesis for less than a year.

4. Patients on antifungal agents.

5. Patients who are not ready to participate in the study.

\section{Sample collection:}

\section{Swab Technique}

Swabs from the tissue surface of the maxillary denture were obtained asfollows:

Samples were obtained in the morning when the patients were fasting, by rubbing a sterile cotton-tipped swab over the palatal impression surface of the maxillary dentures irrespective of the clinical signs for the presence or absence of Candida (Pic.02). It was then cultured on sabouraud dextrose agar (SDA) plates to get pure Candida culture.

The yeast colonies were subcultured onto SDA plates to obtain pure yeast cultures which were harvested, suspended in water in sterile vials and stored at -20 degree $C$. Their phenotypes were defined by culture on CHROM agar Candida plates. (Described below) Further, the colonies were subjected to sugar assimilation and fermentation tests for species identification of Candida. Germ tube assessment was carried out to differentiate C. albicans.

\section{Speciation Of Candida In The Clinical Specimens By Using Chromagar Medium}

In this study speciation was done using HiChrome Candida differential agar. The chromogenic agar plates were prepared as per the manufacturer's instructions. The Candida isolates were inoculated onto the plates and incubated aerobically at 37 degree $C$ for 24 hours. The different species were identified based on the color displayed by them as, Candida albicans (light green) and Candida glabrata (pink) and Candida tropicalis (blue to metallic blue) (Pic.03).

The different species of Candida were first identified by gram staining and later on confirmed by Germ Tube test, Corn Meal agar for chlamydospore formation and other biochemical tests. Chrome Candida differentiation agar was used to differentiate Candida species. Chromagar for 
Candida differentiation uses a chromogenic substance $\beta$ glucosaminidase substrate, which is metabolized to give the colored colonies of different Candida species. HiChrome agar is manufactured as selective and differential media for rapid isolation of yeast and differentiation of candida species by colony color i.e. Candida albicans (light green), C. tropicalis (steel/light blue), C.krusei (white fuzzy) and C. glabrata (pink). Chromagar for differentiation of Candida was prepared following manufacturer's instructions.

\section{Results:}

1. Among the total fifty five denture wearers ( 34 males, 21 females) examined, 27 patients (50\%) were found to be positive for stomatitis.

2. Among the 27 positive patients, 21 were males and 6 were females (Diagram I).

3. Among the total male patients examined, 21patients (61\%) were positive for stomatitis.

4. Among the total female patients examined, 6 patients (28\%) were positive for stomatitis.

5. Among the 27 positive patients, 13 patients (48\%) were positive for $\mathrm{C}$. albicans, 9 patients (33\%) were positive for $\mathrm{C}$. tropicalis, 5 patients (18\%) were positive for C. glabrata. (Diagram II)

\section{Discussion :}

Denture stomatitis has been found to be a common problem associated with denture wearing. The causative agents for this has been ill fitting or poorly fitting complete denture prosthesis causing trauma to the underlying mucosa or improper maintenance protocol by the wearer. In addition, if the patient has suppressed immunity as in Diabetes or HIV, the chances that the normal oral commensal, Candida may convert itself into a pathogen to cause Candida-associated-denture stomatitis (CADS). Approximately 65 to $70 \%$ of the denture wearing population has been identified as afflicted with CADS in other parts of the universe $\mathrm{e}^{1-16}$.

Candida albicans has been the most common causative species in CADS. However, recently it has been reported that there is a shift in this aspect and other species have been found to be superceding $\mathrm{C}$. albicans in causing the infection ${ }^{21,22}$. This is due to development of drug resistance or mutations in the strains causing change in phenotype of the microorganism. In a clinical situation, rapid species level identification is very essential as it has substantial impact on the treatment decisions.

This survey was taken up to assess the prevalence of CADS in this part of the country and also to identify the most frequent causative species related to the condition. From the 55 denture wearing patients who were surveyed it was observed that denture stomatitis was present in 27 patients (50\%), thus proving the occurance of CADS in longterm complete denture wearers is common. Oral samples were collected which were cultured on to SDA medium to obtain pure yeast culture. Once Candida species are separated, it was subjected to speciation procedure using CHROM agar.

Different media have been used to carry out the speciation procedure ${ }^{23,24}$. Among them, CHROM agar has been found to be rapid, accurate and economical. This medium contains a chromogenic $\beta$-glucosaminidase substrate which releases differently colored compounds on degradation by the specific enzymes released by the species. In this medium identification of the species is done by the development of different colors. Candida albicans (light green), C. tropicalis (steel/light blue), C.krusei (white fuzzy) and C. glabrata (light pink). In the present study, it was identified that C. albicans is the most prevalent (50\%) species followed by C. tropicals (33\%) and C. glabrata (18\%).

According to many authors, $\mathrm{C}$. albicans has been the most frequent species of Candida in causing denture stomatitis 2,45,6. Our survey in south west coastal region of Karnataka is in agreement with the same. Cannon et $\mathrm{al}^{2}$ are of the opinion that this yeast has the ability to colonize different areas of the oral cavity due to the specific interactions between the two. It has been known to adhere even to complement receptors and some sugar residues that are present on the host surfaces. Tissue invasion by this species causes infection of the oral mucosa. Parnanen et al 


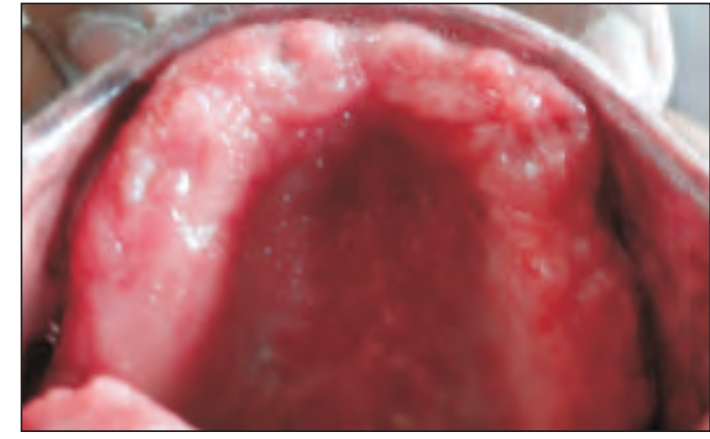

Fig.01 Candida-associated-denture-stomatitis (CADS)

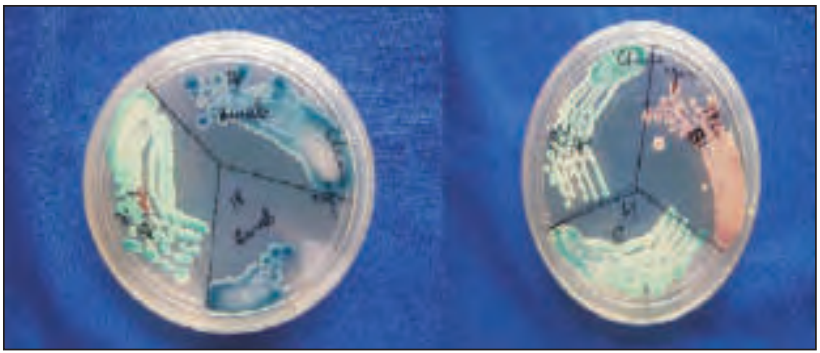

Fig.03 Speciation of Candida

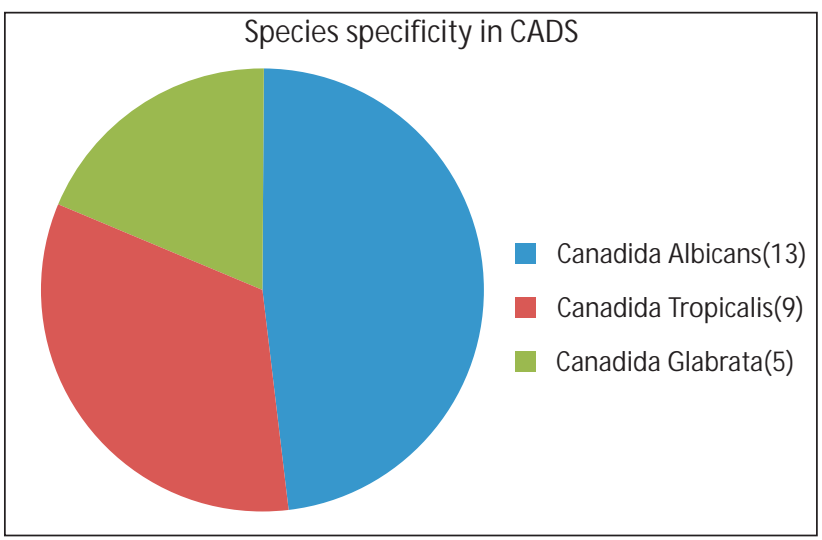

${ }^{26}$ in their comparative study between C. albicans and C. glabrata concluded that C. albicans has the ability to degrade the proteins in both yeast and hyphal forms, where as, C. glabrata did not show this capacity. This shows that $\mathrm{C}$. albicans can invade tissues more easily than $\mathrm{C}$. glabrata making it more prevalent in the oral infections. Salerno et al $^{12}$ said that $C$. albicans can not only adhere to the oral mucosa but also can colonize the surface of the acrylic denture when it is poorly maintained. The biofilm on the denture can enhance its adhesion adding to its virulence. Wearing of a denture has been found to enhance adhesion of $C$. albicans by Witzel et al ${ }^{18,27}$, in the year 2012. However, Williams \& Lewis ${ }^{25}$, are of the opinion that even though $\mathrm{C}$. albicans is the dominant species, it is the problem of emergence of non-Candida-albicans-

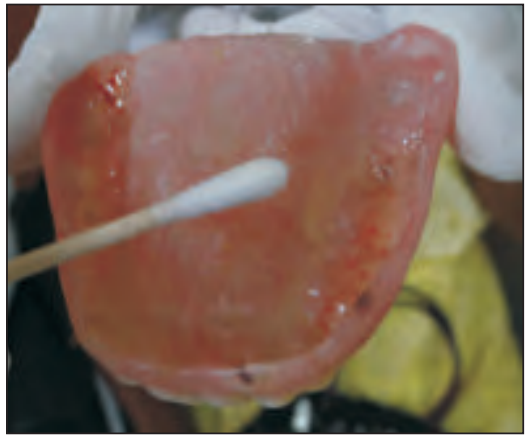

Fig.02 Swab Technique

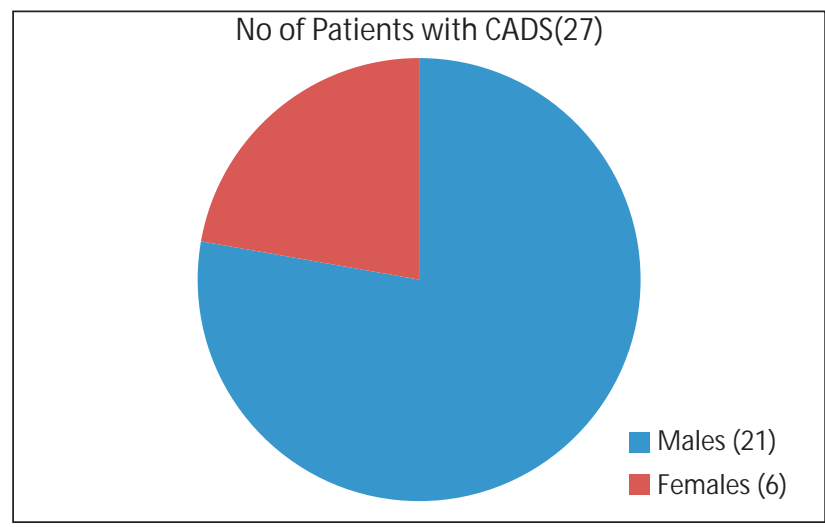

Candida that is problematic in management of the infection. Silva et al ${ }^{20}$ studied the emerging species of Candida in causing stomatitis and concluded that $\mathrm{C}$. glabrata can colonize even after 12 hours and that presence of C. albicans enhances its invasiveness.

Limitations of the study: The risk factors associated with the development of Candida Associated Denture Stomatitis were not considered in this study. Wearing of denture prosthesis is only one of the causes for development of this disease and it is essential to explore the other causes for the problem. Future work should focus on this aspect to identify other etiological factors, both local and systemic, for the occurence of CADS.

\section{Conclusions:}

Within the limitations of this study, it was concluded that

1. Candida associated denture stomatitis is prevalent in denture wearers of South West Coastal region of Karnataka.

2. Males are affected more than females.

3. Candida albicans is the most prevalent species of Candida followed by C. tropicalis and C. glabrata. 


\section{References:}

1. Bulad K, Taylor RL, Verran J, M cCord JF. Colonization and Penetration of Denture Soft Lining M aterials by Candida albicans. Dental M aterials 2004;20:167-75.

2. Cannon RD,Holmes AR,M ason AB,M onk BC. Oral Candida:Clearance, Colonization, or Candidiasis?.J Dent Res 1995;74:1152.

3. Cross $L J$ et al. Evaluation of the Recurrence of Denture Stomatitis and Candida Colonization in a Small Group of Patients who received Itraconazole. Oral Surg Oral Med Oral Pathol Oral Radiol Endod 2004;97:351-8.

4. Dangi YS, Soni ML, Namdeo KP. Oral Candidiasis: A Review. International Journal of Pharmacy and Pharmaceutical Sciences; 2010; 2(4).

5. Dongari-Bagtzoglou A et al. Oral Candida Infection and Colonization in Solid Organ Transplant Recipients. Oral Microbiol Immunol 2009;24:249-54.

6. Liu X, Hua H. Oral Manifestation of Chronic Mucocutaneous Candidiasis: Seven Case Reports. J oral Pathol Med 2007;36:528-32.

7. Milillo Let al. Candida-Related Denture Stomatitis: A Pilot Study of the Efficacy of an Amorolfine Antifungal Varnish. Int J Prosthodont 2005;18:55-59.

8. Olczak-Kowalczyk D et al. Oral Candidiasis in Immunosuppressed Children and Young Adults After Liver or Kidney Transplantation. Pediatr Dent 2010;32:189-94.

9. Pereira-cenci $T$, Del bel cury AA, Crielaard W, Ten cate JM Development of Candida-associated Denture Stomatitis: New Insights. J Appl Oral Sci. 2008;16:86-945.

10. Perezous LF, Flaitz CM, Goldschmidt ME, Engelmeier RL. Colonization of Candida species in denture wearers with emphasis on HIV infection: A literature review. J Prosthet Dent 2005;93:288-93.

11. Reichart PA, Samaranayake LP, Bendick Ch, Schmidt-Westhausen M Jayalathike MS. Prevalence of Oral Candida Species in Leprosy Patients from Cambodia and Thailand. J Oral Pathol Med 2007;36: 342-6.

12. Salerno C et al. Candida-associated Denture Stomatitis. Journal section: Oral Medicine and Pathology.(Ahead of print): 2010.

13. Scully C,El-Kabir M, Samaranayake LP. Candida and Oral Candidosis: A Review. Critical Reviews in Oral Biology and M edicine,1994;5:125-157.

14. Thompson GR et al. Oropharyngeal candidiasis in the era of antiretroviral therapy. Oral Surg Oral Med Oral Pathol Oral Radiol endod 2010;109:488-95.
15. Webb BC, Thomas C], Willcox M DP, Harty DWS, Knox KW. Candidaassociated Denture Stomatitis. Aetiology and M anagement: A review Part 3. Treatment of oral candidosis. Australian Dental Journal 1998;43:000-000

16. Zunt SL. Oral Candidiasis: Diagnosis and Treatment. The J of Practical Hygiene 2000;31-36.

17. Ramage $\mathrm{G}$ et al. Denture Stomatitis: A role for Candida biofilms. Oral Surg Oral M ed Oral Pathol Oral Radiol Endod 2004;98:53-9.

18. Emami e, Taraf H, Grandmont P, Gauthier G, Koninck L, Lamarche C, Souza RF. The Association of Denture Stomatitis and Partial Removable Dental Prostheses: A Systematic Review. Int J Prosthodont 2012;25:113-119

19. Sanita PV, Pavarina AC, Giampaolo ET, Silva M M, M ima EGO, Ribeiro DG, Vergani CE. Candida spp. Prevalence in well controlled type 2 diabetic patients with denture stomatitis. Oral Surg Oral Med Oralpathol Oral Radiol Endod 2011;111:726-33.

20. Silva S, Henriques M, Hayes A, Oliveira R, Azeredo J, Williams DW. Candida glabrata and Candida albicans co-infection of an in vitro ora epithelium. J Oral Pathol Med. 2011;40:421-27.

21. Richardson MD. Changing patterns and trends in systemic fungal infections. J of Antimicrobial Chemotherapy 2005;56:Suppl.S1,i5-i11.

22. Jabra-Rizk M A et al. Fungal biofilms and drug resistance. Emerg Infect Dis. 2004;10:14-9.

23. Nadeem SG, Hakim ST, Kazmi SU.Use of CHROM agar Candida for the presumptive identification of Candida species directly from clinical specimens in resource-limited settings. Libyan J Med 2010, 5: 2144 DOI: 10.3402/ljm.v5i0.2144, pg.1-6

24. Yicesoy M , M arol S. Performance of CHROM AGAR candida and BIGGY agar for identification of yeast species.Annals of Clinical M icrobiology and Antimicrobials 2003, 2:8, pg.1-7

25. Williams D, Lewis M. Pathogenesis and treatment of oral candidosis. Journal of Oral M icrobiology 2011:3.

26. Parnanen P, M eurman JH, Samaranayake L, Virtanen I. Human Oral Keratinocyte E-cadherin Degradation by Candida albicans and Candida glabrata. J Oral Pathol Med 2010;39:275-78

27. Witzel AL, Pires M FC, Carli M L, Rabelo GD, Nunes TB. Candida albicans isolation from Buccal M ucosa of Patients with HIV wearing removable dental prostheses. Int J Prosthodont 2012;25:127-131. 